\title{
EDITORIAL
}

\section{Why does the United States need a national center for new cures?}

\author{
Molecular Psychiatry (2011) 16, 882-884; doi:10.1038/ \\ mp.2011.103
}

A new National Center for Advancing Translational Sciences (NCATS) has been proposed to the United States Congress as a component of the National Institutes of Health (NIH). Much debate has ensued as to whether a new center, tentatively budgeted at a cost of one billion dollars a year, is necessary at a time of budgetary constraint. What has been missed in the discussion that has originated around this proposal is the unrecognized fact that it represents a paradigm shift. The mission of NCATS moves the focus of translational medicine away from the domain of infrastructure to that of large-scale, mission-driven discovery in order to specifically and cohesively target the developments of new treatments and cures.

Paraphrasing Charles Dickens, this is the best of times, this is the worst of times. Beginning in 1998, the annual budget of the NIH doubled over 5 years, and it is currently $\$ 31$ billion. This efflux of resources together with powerful and increasingly cheaper technologies have generated a revolution in medical research. The amount of fundamental discovery is staggering, and medical journals are choked with spectacular science. As editor of Molecular Psychiatry, the highest-ranking journal in its field, I go over 1000 papers per year, but can only publish $3 \%$ of submissions. The avalanche of outstanding research is overwhelming. That being the case, why is this the worst of times?

Paradoxically, research advances have not led to new cures. Most scientific papers or NIH grants comment on the potential impact of the research on health, but new treatments or cures fail to materialize. My own areas of research are obesity and major depression. Two-thirds of Americans are overweight or obese. ${ }^{1}$ Obesity is a public health time bomb, ticking itself away toward catastrophe. All the progress in public health accomplished in the last few decades, leading to increased health and longevity, may be obliterated by the long-term negative impact of obesity. In the last 20 years, there has been an explosion in the science underlying the genetics, basic biology and brain circuits regulating food intake and satiety, resulting in obesity. ${ }^{2,3}$ Yet, such knowledge has not been translated to any new drugs that decrease weight safely and effectively. The cost of obesity to the US economy is $\$ 150$ billion yearly. ${ }^{4}$ The economic burden of depression is $\$ 100$ billion annually. ${ }^{5}$
Depression is the second cause of disability. Suicide, which is mostly a consequence of depression, is the eleventh overall cause of death; it is the third cause of death in the age group of 15-24 years and the fourth cause of death in the age group of 25-44 years. ${ }^{6}$ Most depressed patients are treated with antidepressants such as the selective serotonin reuptake inhibitors. Antidepressants represent the second most widely sold class of drugs in America, the first one being analgesics. The action of the majority of antidepressants is based on a scientific principle elucidated by Nobel laureate Julius Axelrod in 1961, the reuptake of monoamines such as norepinephrine and serotonin by brain neurons. ${ }^{7}$ Ketamine is being investigated as an experimental treatment for depression, ${ }^{8}$ yet the first use of this drug in humans was reported in 1965 . The myriad scientific discoveries of the last 40 years have not helped patients with depression. The lack or paucity of new treatments is not restricted to obesity or depression-it cuts across most human diseases.

This grievous gap from discovery to cure has been called by experts 'the valley of death' or 'the cesspool of devastation'. ${ }^{9}$ The medical establishment has appropriately recognized the urgent need for what has been named 'translational science,' aimed at facilitating the pathway from discovery to health. The establishment of translational science as an emerging area of medicine is positive, as it creates a workforce that is specialized in bridging the lab and the clinic and it establishes pipelines for this transition. The NIH is already funding, through its Clinical and Translational Science Awards (CTSAs), 60 large translational centers located across the country at an annual cost of $\$ 500$ million to provide outstanding academic pipelines for translation of research to healthcare. Importantly, the pharmaceutical industry is a major stakeholder because it is directly invested in the outcomes of new treatments.

Yet, Francis Collins, the NIH Director, now proposes a new NIH center, the NCATS. This initiative has been faced with considerable controversy and intense questioning as to whether this new center, costing approximately one billion dollars per year, is really needed. ${ }^{10}$

I believe that the answer is 'yes', but for a reason that has not been previously discussed. Implicit in the creation of NCATS is a revolutionary paradigm shift in translational medicine from infrastructure to mission-driven discovery. Existing efforts such as the CTSA initiative are focused on creating highly 
complex structures to translate science to the clinic. Our current assumption is that discoveries are just out there or will emerge from the work of individual scientists who are guided by their curiosity, and then we can simply develop a bridge to the clinic. That does not work and has not worked so far. Research driven by individual scientists can truly advance knowledge and should be supported, but it will not resolve large-scale, complex problems that go across multiple domains of science. Putting a man on the moon or sequencing the entire human genome required well-coordinated, mission-driven efforts that brought together experts of the diverse backgrounds over very long period of times that far exceeded the 3-5 year research grants to individual researchers.

Current efforts in translational medicine are akin to having a large group of capable but disconnected experts individually planning and building exquisite and expensive bridge structures without central coordination and disregarding the origins of traffic flow patterns. What is currently proposed is a highly innovative paradigm shift on the emphasis of medical translation from infrastructure to mission-driven discovery. Although infrastructure is important, and should be funded, the proposed NCATS will be specifically charged with discovering new treatments and cures, which is what the taxpayers want and need when they, through their elected representatives, finance NIH at a tune of $\$ 31$ billion every year. In the absence of new funding, resources for NCATS may have to come from existing NIH-funded areas and there is naturally an outcry from stakeholders of such programs. That is expected, but no priority should be higher at the NIH than discovery that leads to cures-or at least to better treatments.

Another group of skeptics says that finding cures should be the exclusive domain of drug companies. I disagree. The pharmaceutical industry's pipeline is bone dry. Drug development has become very risky: the focus of publically held companies are the shareholders and their short-term profit, not longterm research that could lead to new cures. Having the NIH develop a novel therapeutic pipeline, which would be patented by the government, then passed on to companies for less risky, less costly and more expeditious development, would greatly increase the number of available new treatments. Over time, this process might bring revenue back to $\mathrm{NIH}$, leading to a positive cycle of increased resourcing toward the development of new treatments and cures.

Some may be miffed at the possibility of the government obtaining patents and potentially earning revenue from fundamental drug discovery. Those should be reminded that patenting is the bedrock of translation. No pharmaceutical company will develop extensive and expensive phase 2 and 3 trials for compounds that are in the public domain. The absence of patents for the underlying fundamental basis of treatments assures their lack of commercial potential and undermines translational prospects.
Therefore, the NIH would need to rapidly patent promising leads emerging from NCATS and to license them in a fair and transparent manner that is compatible with the best interests of the American taxpayers, who will be funding NCATS. NCATS will be a small component of NIH and an even smaller component of the nation's overall drug development effort. However, small enterprises can be highly catalytic and have major impact, particularly if the new center becomes the organizational headquarters and launching pad for conceptually novel therapeutic discovery efforts that are pursued as governmentindustry-academia partnerships. It will be a challenge for NCATS to achieve these goals in the context of NIH's strict conflict of interest policies; therefore, one of the first strategic goals for NCATS ought to be the development of clear guidelines for interactions with other sectors of government, such as the Food and Drug Administration, as well with academia, not-for-profit entities and for-profit companies, all the way from small startups to big pharma.

The creation of NCATS as proposed by Francis Collins is truly needed. Its final configuration will certainly evolve over time as the new entity grows and develops relationships both with other sectors of government as well as with industry. A very strong community advisory board would be highly desirable right from the outset to guide NCATS's complex external relations as well as to discuss priority settings.

In a recent article ${ }^{11}$, Francis Collins presented his initial vision and strategic plan for NCATS as a 'catalytic hub.' According to him, 'early discussions with a variety of stakeholders have identified several components of translational science that are ripe for the new scientific approach offered by NCATS and will likely be the subject of early targeted funding opportunities.' These include the following key themes:

- Therapeutic target validation

- Chemistry

- Virtual drug design

- Preclinical toxicology

- Biomarkers

- Efficacy testing

- Phase zero clinical trials (defined as very preliminary studies using as few as one or two human volunteers)

- Rescuing and repurposing

- Clinical trial design

- Post-marketing research

Current plans for NCATS $^{11}$ include the following initial constitutive elements:

- CTSA Program: These are infrastructure grants awarded to academic medical institutions to facilitate translational research. They represent a network of 60 US centers, with expertise in preclinical science, clinical trials, comparative effectiveness research, training and community engagement. 
- Components of the Molecular Libraries Program: This supports the centers that provide access to large-scale screening, medicinal chemistry and informatics for the identification of therapeutic and experimental chemical entities. They provide assay development, high-throughput screening, medicinal chemistry and compound databases.

- Therapeutics for Rare and Neglected Diseases (TRND): A drug-development pipeline within the NIH used for research collaborations with academic scientists, non-profit organizations and companies working on rare and neglected illnesses. TRND offers preclinical development of promising compounds.

- Rapid Access to Interventional Development (RAID): This is a competitive granting program that provides resources for the development of new therapeutic agents and provides access to resources for preclinical development, production, bulk supply, GMP manufacturing, formulation, development of an assay suitable for pharmacokinetic testing and animal toxicity.

- Office of Rare Diseases Research: A multifunctional NIH office that serves as a focal point for rare diseases and offers coordination and support of research on rare diseases.

- The NIH-FDA Regulatory Science Initiative: A competitive grant program that funds regulatory science and supports research on applicability of novel technologies and approaches to regulatory review of drugs, biologics and devices.

- Cures Acceleration Network (CAN): This is new, competitive grant program to fund translational solutions to high-need medical problems; it awaits appropriation. It was designed to prove support of translational research with greater flexibility to NIH to fund innovative research in therapeutic development.

Put together, these programs together currently have $\sim \$ 720$ million annually in research support, without including CAN, which has not yet been yet established and funded by Congress.

Collins's efforts leading the International Human Genome Sequencing Consortium were initially criticized as too expensive, overly ambitious and a drain on resources that could go to smaller projects initiated by individual scientists. The sequencing of human genome with results that are freely available to all is now a hallmark of contemporary science. Creating a mission-driven center at the NIH to foster new cures is even more visionary. The focus of translational medicine must shift from infrastructure building to a cohesive and mission-driven effort toward the discovery of more effective treatments and new cures. NCATS is well poised to be the place for this to happen.

\section{Conflict of interest}

The authors declare no conflict of interest.

J Licinio and M-L Wong

Department of Translational Medicine, John Curtin School of Medical Research, Australian National University, Canberra, ACT, Australia E-mail: julio.licinio@anu.edu.au

\section{References}

1 Nguyen DM, El-Serag HB. The epidemiology of obesity. Gastroenterol Clin North Am 2010; 39: 1-7.

2 Li S, Zhao JH, Luan J, Luben RN, Rodwell SA, Khaw KT et al. Cumulative effects and predictive value of common obesitysusceptibility variants identified by genome-wide association studies. Am J Clin Nutr 2010; 91: 184-190.

3 Baicy K, London ED, Monterosso J, Wong ML, Delibasi T, Sharma A et al. Leptin replacement alters brain response to food cues in genetically leptin-deficient adults. Proc Natl Acad Sci U S A 2007; 104: 18276-18279.

4 Finkelstein EA, Trogdon JG, Cohen JW, Dietz W. Annual medical spending attributable to obesity: payer-and service-specific estimates. Health Aff (Millwood) 2009; 28: w822-w831.

5 Greenberg PE, Kessler RC, Birnbaum HG, Leong SA, Lowe SW, Berglund PA et al. The economic burden of depression in the United States: how did it change between 1990 and 2000? J Clin Psychiatry 2003; 64: 1465-1475.

6 Milane MS, Suchard MA, Wong ML, Licinio J. Modeling of the temporal patterns of fluoxetine prescriptions and suicide rates in the United States. PLoS Med 2006; 3: e190.

7 Hertting G, Axelrod J. Fate of tritiated noradrenaline at the sympathetic nerve-endings. Nature 1961; 192: 172-173.

8 Li N, Lee B, Liu RJ, Banasr M, Dwyer JM, Iwata M et al. mTORdependent synapse formation underlies the rapid antidepressant effects of NMDA antagonists. Science 2010; 329: 959-964.

9 Licinio J. Translational Psychiatry: Leading the transition from the cesspool of devastation to a place where the grass is really greener. Translational Psychiatry 2011; 1, (e1; doi:10.1038/tp.2011.3).

10 Kaiser J. US science policy. Collins sparks furor with proposed NIH reshuffling. Science 2011; 331: 386.

11 Collins FS. Reengineering Translational Science: the time is right. Sci Transl Med; Published online 6 July 2011, 3: 90cm17. 